\title{
Emotional Facial Expressions Modulate Pain-Induced Beta and Gamma Oscillations in Sensorimotor Cortex
}

\author{
Daniel Senkowski, ${ }^{1,2}$ Janine Kautz, ${ }^{1}$ Michael Hauck, ${ }^{1,3}$ Roger Zimmermann, ${ }^{1}$ and Andreas K. Engel ${ }^{1}$ \\ ${ }^{1}$ Department of Neurophysiology and Pathophysiology, University Medical Center Hamburg-Eppendorf, 20246 Hamburg, Germany, ${ }^{2}$ Department of \\ Psychiatry and Psychotherapy, Charité University Medicine Campus Mitte, St. Hedwig Hospital, 10115 Berlin, Germany, and ${ }^{3}$ Department of Neurology, \\ University Medical Center Hamburg-Eppendorf, 20246 Hamburg, Germany
}

Painful events in our environment are often accompanied by stimuli from other sensory modalities. These stimuli may influence the perception and processing of acute pain, in particular when they comprise emotional cues, like facial expressions of people surrounding us. In this whole-head magnetoencephalography (MEG) study, we examined the neuronal mechanisms underlying the influence of emotional (fearful, angry, or happy) compared to neutral facial expressions on the processing of pain in humans. Independent of their valence, subjective pain ratings for intracutaneous inputs were higher when pain stimuli were presented together with emotional facial expressions than when they were presented with a neutral facial expression. Source reconstruction using linear beamforming revealed pain-induced early (70-270 ms) oscillatory beta-band activity (BBA; $15-25 \mathrm{~Hz}$ ) and gamma-band activity (GBA; $60-80 \mathrm{~Hz}$ ) in the sensorimotor cortex. The presentation of faces with emotional expressions compared to faces with neutral expressions led to a stronger bilateral suppression of the pain-induced BBA, possibly reflecting enhanced response readiness of the sensorimotor system. Moreover, pain-induced GBA in the sensorimotor cortex was larger for faces expressing fear than for faces expressing anger, which might reflect the facilitation of avoidance-motivated behavior triggered by the concurrent presentation of faces with fearful expressions and painful stimuli. Thus, the presence of emotional cues, like facial expressions from people surrounding us, while receiving acute pain may facilitate neuronal processes involved in the preparation and execution of adequate protective motor responses.

\section{Introduction}

Facial expressions of people surrounding us provide the emotional context in most social situations and may therefore influence the processing of pain. The perception of emotional facial expressions modulates neuronal activity in the limbic system (Adolphs, 2002) and also elicits rapid and widely automatic responses in other brain areas (Batty and Taylor, 2003; Frith, 2009). Moreover, the cortical regions involved in the decoding of pain (Bushnell et al., 1999) and emotional facial expressions (Adolphs et al., 2000), like the somatosensory cortex, partly overlap. For these reasons, it is likely that emotional facial expressions modulate the processing of pain.

Emotional cues, such as pictures with affective content (Godinho et al., 2006) or pleasant (e.g., floral scents) or unpleasant odors (Villemure and Bushnell, 2009), influence pain processing.

\footnotetext{
Received Nov. 16, 2010; revised July 11, 2011; accepted Aug. 8, 2011.

Author contributions: D.S., M.H., R.Z., and A.K.E. designed research; D.S., J.K., and R.Z. performed research; R.Z. contributed unpublished reagents/analytic tools; D.S., J.K., and M.H. analyzed data; D.S., M.H., and A.K.E. wrote the paper.

This study was supported by grants from the Deutsche Forschungsgemeinschaft (SE 1859/1-2, D.S.; SFB TRR 58 B04, A.K.E.) and the European Union (ERC-2010-StG_20091209, D.S.; IST-2005-27268, NEST-PATH-043457, HEALTH-F2-2008-200728, ERC-2010-AdG-269716, A.K.E.). We thank K. Saha and S. Scheer for recruitment of participants and help with data recordings and Constanze Hipp and Christoph Kayser for their comments on the manuscript.

Correspondence should be addressed to Dr. Daniel Senkowski, Department of Neurophysiology and Pathophysiology, University Medical Center Hamburg-Eppendorf, Martinistrasse 52, 20246 Hamburg, Germany. E-mail: dsenkows@uke.uni-hamburg.de.

DOI:10.1523/JNEUROSCI.6002-10.2011

Copyright $\odot 2011$ the authors $\quad 0270-6474 / 11 / 3114542-09 \$ 15.00 / 0$
}

Compared to control stimuli, sensory inputs that induce positive emotion often lead to analgesic effects, i.e., reduction of pain perception (Meagher et al., 2001). In contrast, pain ratings of painful stimuli that are presented with inputs that induce negative emotions are frequently enhanced (Godinho et al., 2006). Interestingly, a recent study showed that both pleasant (i.e., lemon scent) and unpleasant odors lead to enhanced pain ratings (Martin, 2006), suggesting that the effect of positive emotional inputs on pain processing may depend on the type of stimulus (e.g., type of odor) used to induce positive emotions. To date, the impact of emotional facial expressions on pain perception and the neuronal mechanisms underlying the crossmodal influence of facial expression on the processing of pain are not understood.

A neuronal mechanism that may be crucial for the impact of facial expression on the processing of pain is synchronization of neuronal populations. Recent studies suggested that neuronal synchronization, in particular in higher-frequency betaband activity (BBA; $15-30 \mathrm{~Hz}$ ) and gamma-band activity (GBA; activity above $30 \mathrm{~Hz}$ ), plays an important role for crossmodal processing (Senkowski et al., 2006, 2008; Lakatos et al., 2007; Kayser and Logothetis, 2009). Recently, neural synchronization mechanisms were also linked to pain processing and pain perception (Hauck et al., 2008). Specifically, the GBA in primary somatosensory (Gross et al., 2007; Tiemann et al., 2010) and sensorimotor cortex (Hauck et al., 2007) was positively correlated to pain perception. Furthermore, it has been shown that painful inputs suppress oscillatory activity in the alpha-band (7-15 Hz) and beta-band (Ploner et al., 2006a). 
This suggests that oscillatory responses, in particular in regions of the sensorimotor cortex, are an important neuronal mechanism for the encoding of pain.

In this magnetoencephalography (MEG) study, we investigated the influence of emotional facial expressions on pain processing, with a focus on higher-frequency oscillatory activity in the sensorimotor cortex. Using linear beamforming (Gross et al., 2001), pain-induced oscillatory responses were examined for faces with positive (happiness) and negative (fear, anger) valence and compared with the responses to neutral facial expressions. Independent of the valence of the facial expression, subjective pain ratings were higher for emotional than for neutral expressions, an effect that was paralleled by modulations in early pain-induced BBA in the sensorimotor cortex. Additionally, differences in the early pain-induced GBA were found between the facial expressions of fear and anger.

\section{Materials and Methods}

Participants. Nineteen right-handed healthy volunteers participated in the experiment (mean age 24 years, range $=21-28$ years, 8 female). One additional participant was excluded due to technical artifacts during the MEG recording. All participants had normal or corrected-to-normal vision and reported no history of neurological or psychiatric illness. The review board of the Hamburg Medical Association's Ethics Commission approved the study, and each subject provided written informed consent.

Stimuli. The intracutaneous pain model (Bromm and Meier, 1984) was used to induce pain. Electrical stimulus pulses (16 ms duration) were delivered to the nearest proximity of nociceptors using a thin electrode that was fed through a hole inserted into the epidermal skin at the tip of the left middle finger. Before the experiment, each participant's individual pain threshold was determined by applying a staircase method with successive intensity increments and decrements of $0.02 \mathrm{~mA}$. The individual threshold of a participant was computed as the average intensity at which the participant reported a given stimulus as painful. During the experiment, standard stimuli were applied with an intensity 1.5 -fold of the individual pain threshold in $90 \%$ of all painful inputs (i.e., in P-only and bimodal PV trials; see below, Procedure). In addition, $10 \%$ of all painful stimuli were presented at twofold of the individual pain threshold. These stimuli, which served as catch trials, were presented to avoid a possible tendency of participants to rate all trials equally.

Visual stimuli comprised a set of pictures from the Ekman FEEST stimulus battery (Young et al., 2002). Faces of eight actors (four female) expressing three emotions (fear, anger, and happiness) and neutral control expressions were presented. The pictures of the faces were displayed at a visual angle of $10.8^{\circ}$ in the horizontal plane and $15.2^{\circ}$ in the vertical plane. Stimuli were projected from a calibrated LCD projector outside of the magnetically shielded chamber at a refresh rate of $60 \mathrm{~Hz}$. Stimulus presentation was controlled using "Presentation" software (Neurobehavioural Systems).

Procedure. Participants were presented with a continuous randomized stream of visual face stimuli (V-only), pain stimuli (P-only), and bimodal pain-visual (PV) stimuli (Fig. 1a). They were instructed to attend to the painful intracutaneous stimuli and to rate the perceived unpleasantness of these stimuli on a visual analog scale (VAS; range 1-100, where 1 indicates a very low and 100 a very high pain experience), independent of whether they were presented alone or in combination with a task-irrelevant face. Moreover, while maintaining central fixation, participants were asked not to pay particular attention to the faces. Unpleasantness ratings, which are a subjective measure of the affectivemotivational dimension of the nociceptive experience (Price, 2000), have been previously shown to reflect emotional modulations of pain (Villemure and Bushnell, 2009). Visual and pain inputs had the same onsets in the PV trials. One thousand milliseconds after the onset of the P-only and PV stimuli, the VAS was presented for $1200 \mathrm{~ms}$, during which the participants rated the unpleasantness of the painful inputs using a joystick in their right hand. A fixed interval of $400 \mathrm{~ms}$ followed the offset of $\mathrm{V}$-only stimuli, which were presented for $600 \mathrm{~ms}$. In addition, an inter-
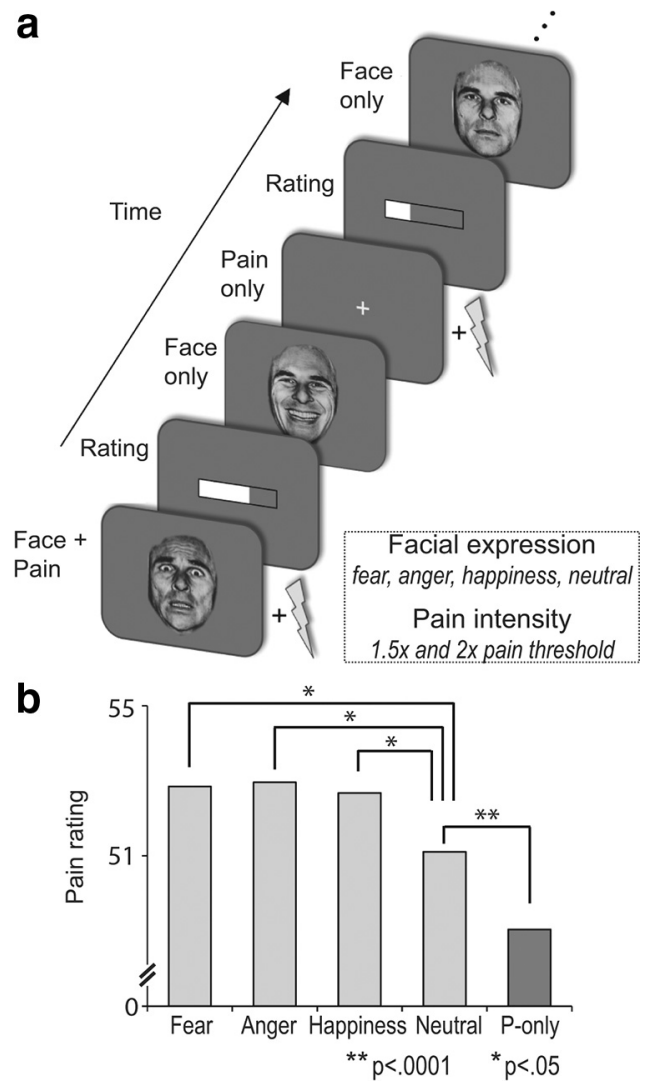

Figure 1. Experimental setup and behavioral data. $\boldsymbol{a}$, Participants were presented with a continuous stream of faces only, pain only, and bimodal PV stimuli. They were instructed to rate the painful events on a visual analog scale. Faces with three different emotions (fear, anger, and happiness) and neutral control expressions were presented. $\boldsymbol{b}$, Pain ratings to standard trials were higher for the three emotional facial expression conditions than for the neutral control condition. Moreover, pain ratings were significantly higher when a face was presented simultaneously than in pain only trials.

stimulus interval of $800-1200 \mathrm{~ms}$ (mean $1000 \mathrm{~ms}$ ) followed the presentation of each stimulus. A central fixation cross was presented during periods in which no face or VAS was shown. In total (standard and catch trials), $300 \mathrm{P}$-only, $600 \mathrm{~V}$-only (150 for each facial expression type), and 600 bimodal PV (150 for each facial expression type) inputs were presented. The order of stimuli was randomized with the limitation that not more than two faces with the same expression were shown consecutively. The experiment comprised 15 blocks, each of which lasted $\sim 4.5 \mathrm{~min}$. In addition, one practice block was conducted to familiarize the participants with the experimental procedure.

$M E G$ recordings and data preprocessing. The MEG was recorded in a magnetically shielded chamber using a 275-channel whole-head system (Omega 2000, CTF Systems). Three defective MEG channels were removed from further analysis. Simultaneous to the MEG recording, eye movements and eye blinks were monitored using two electrooculogram electrodes for the offline artifact rejection. The head position of participants was measured before and after each experimental block. In the vast majority of blocks, the head displacements were $<2 \mathrm{~mm}$, and for all analyzed datasets, the head displacements were $<10 \mathrm{~mm}$. MEG data were low-pass filtered online (cutoff $300 \mathrm{~Hz}$ ) and recorded at a sample rate of $1200 \mathrm{~Hz}$. Analysis of MEG data was performed using Matlab (MathWorks) and FieldTrip (http://www.ru.nl/fcdonders/fieldtrip). For data reduction, the recorded signals were offline high-pass filtered at $2 \mathrm{~Hz}$ and low-pass filtered at $170 \mathrm{~Hz}$. Moreover, data were resampled at $400 \mathrm{~Hz}$. To remove line-noise artifacts, notch filters at 50,100, and $150 \mathrm{~Hz}$ (bin width $=1 \mathrm{~Hz}$ ) were used. Data were epoched from $-800 \mathrm{~ms}$ before to $2400 \mathrm{~ms}$ after the onsets of V-only, P-only, and bimodal PV events. Trials containing eye blinks, eye movements, muscle artifacts, or signal jumps 
were rejected manually from further analysis. In addition, bad channels were offline interpolated by replacing them with the average of the neighboring channels. Specifically, two non-neighboring channels in one dataset and three non-neighboring channels in another dataset were interpolated.

Analysis of oscillatory responses. Time-frequency representations (TFRs) were computed using the multitaper method applied to short sliding time windows. This method offers an optimal spectral concentration over the frequency range of interest (Mitra and Pesaran, 1999). The data in each time window were multiplied with a set of orthogonal Slepian tapers. The Fourier transforms of the tapered time windows were then calculated and the resulting power estimates were averaged across tapers. For the examination of low-frequency $(2-30 \mathrm{~Hz})$ and highfrequency $(30-120 \mathrm{~Hz})$ activities, fixed time windows $(\Delta T=200 \mathrm{~ms}$ and $\Delta T=150 \mathrm{~ms}$, respectively) and fixed frequency smoothing $(\Delta f= \pm 5 \mathrm{~Hz}$ and $\Delta f= \pm 10 \mathrm{~Hz}$, respectively) were applied, resulting in one taper for the low frequency range and two tapers for the high frequency range. For the calculation of total power (i.e., activity that comprises both evoked and induced oscillations), frequency transformation was performed on the single-trial level separately for each frequency before averaging for frequencies from 2 to $120 \mathrm{~Hz}(0.5 \mathrm{~Hz}$ frequency step size and $5 \mathrm{~ms}$ window step size). To reveal amplitude-normalized signal changes of total power in the poststimulus interval, the average power in the baseline interval ( -300 to $-100 \mathrm{~ms}$ before stimulus onset) was first subtracted and the resulting difference was divided by the baseline interval activity for each frequency as follows: $\operatorname{Pow}(t, f)_{\text {normalized }}=100 \times\left(\operatorname{Pow}(t, f)_{\text {poststimulus }}-\right.$ $\left.\operatorname{Pow}(f)_{\text {baseline }}\right) / \operatorname{Pow}(f)_{\text {baseline. }}$

A linear beamforming approach was applied for the source reconstruction of total oscillatory responses (Van Veen et al., 1997; Gross et al., 2001). In short, this approach uses an adaptive spatial filter that passes activity from one specific location of interest with unit gain and maximally suppresses other activity. Since linear beamforming is based on the calculation of the cross-spectral density matrix over trials, this approach is particularly suitable for the analysis of total power in the human electroencephalogram (Schneider et al., 2008, 2011) and magnetoencephalogram (Bauer et al., 2006; Donner et al., 2009). The spatial filters for the beamforming analysis were computed based on pooled trials across the four facial expressions (separately for V-only and bimodal PV trials and separately for baseline and poststimulus intervals). The use of common filters ensures that differences in source space activity can be ascribed to power differences in the different facial expressions and not to differences between filters. Single trials were then projected through these filters and averaged in source space separately for each condition.

For the source reconstruction, T1-weighted structural MRIs were recorded on a 3 tesla MRT individually for all participants and single-shell models were derived from the segmentation of MRIs using the Statistical Parametric Mapping software (SPM2; http://www.fil.ion.ucl.ac.uk/ $\mathrm{spm})$. The lead field matrix was calculated on a boundary element model (BEM) for each grid point in the brain with a regular $7 \mathrm{~mm}$ grid. The source activity at each grid point was estimated by constructing a spatial filter using the lead field at this point and the cross-spectral density matrix. The cross-spectral density matrix was calculated between all MEG channels separately for a baseline time interval (centered at -200 $\mathrm{ms}$ ) and for those poststimulus time points and frequencies in the betaband and gamma-band for which robust amplitude changes were observed (Figs. 2, 3). To examine oscillatory responses in source space across participants, a BEM template grid was first created based on the template brain from the Montreal Neurological Institute (MNI; http:// www.mni.mcgill.ca). Subsequently, the individual BEM head models were warped into the template grid and the inverse of that warp was applied to the individual grids. Due to the warping, a specific grid point (in MNI coordinates) is located in the same area of the brain across all participants. Since the most robust beta-band and gamma-band responses were observed in the ipsilateral and the contralateral sensorimotor hand region (Fig. 2), a symmetric hand region comprising sensorimotor cortex was used for the region of interest (ROI) analysis of total oscillatory activity (spatial coordinates for determining the ROI were taken from http://neuro.imm.dtu.dk/services/jerne/ninf/voi/hand_area.html).
For the study of effects on evoked power (i.e., oscillatory responses that are strictly phase locked across trials), the transformation to the frequency domain was performed on the average of all trials, i.e., the event-related response, separately for each frequency and condition. Evoked responses were calculated by subtracting the baseline interval activity from the poststimulus interval activity without further dividing these responses by the baseline (since evoked power is expected to be very low in the prestimulus interval). Moreover, the analysis of effects in evoked oscillatory responses was confined to the sensor level using two regions of interest that comprised six sensors each. The sensors were selected in accordance with the main activity patterns found in total oscillatory responses, which were localized to the sensorimotor hand region (Fig. 2a). Since there is no standard position for MEG sensors relative to the head position across participants, data of each participant were realigned to a standard helmet before the analysis of evoked responses. The procedure used has been described previously (Knosche, 2002).

Statistical analysis. To examine whether pain ratings of non-catch trials were normally distributed, Shapiro-Wilk parametric tests were conducted within each participant and condition. If these tests indicated violations of normal distribution, median values of pain ratings were submitted to further analysis. In line with the activity pattern of paininduced total oscillatory responses (Figs. 2,3), which are to a large extent comparable with our previous study (Hauck et al., 2007), the focus of the statistical analysis of oscillatory responses was on the examination of BBA $(13-23 \mathrm{~Hz})$ and GBA $(70-90 \mathrm{~Hz})$ in the hand region of the sensorimotor cortex. An early time interval centered at $170 \mathrm{~ms}$ was subjected to the statistical analysis. The motivation for studying effects at this interval derives from electrophysiological studies on classical evoked brain responses (Ploner et al., 1999; Nakata et al., 2008). These studies suggested critical nociceptive processes in a cortical network including sensorimotor areas at this latency. Furthermore, neuronal activity around $170 \mathrm{~ms}$ has been closely related to the encoding of emotional facial expressions (Batty and Taylor, 2003; Eimer and Holmes, 2007). Therefore, it is possible that emotional facial expressions modulate pain-induced oscillatory responses around $170 \mathrm{~ms}$. In the present study, robust modulations of the early BBA and GBA were observed. These modulations were paralleled by an increase in lower frequency total activity around $200 \mathrm{~ms}$. For exploratory purposes, effects of emotional facial expressions on paininduced lower frequency activity $(3-9 \mathrm{~Hz}$, centered at $170 \mathrm{~ms}$ ) were examined (see Notes).

The statistical data analysis comprised two levels. The first level of analysis was conducted to investigate valence-specific effects of facial expressions on pain-induced oscillatory responses. For this analysis, the responses to V-only stimuli were subtracted from the responses to the respective visual inputs that were presented in the bimodal context. For instance, the response to a facial expression of fear (i.e., $V_{\text {Fear }}$ ) was subtracted from the response obtained for the same facial expression that was presented in combination with a painful stimulus (i.e., $\left.\mathrm{PV}_{\mathrm{Fear}}\right)$. This subtraction effectively eliminated response differences, which are solely driven by the visual inputs, leaving pain-induced oscillatory responses in the context of the crossmodal bias of the different facial expressions. For the analysis of evoked oscillatory responses, the response differences between PV and V-only stimuli were computed before the time-frequency transformation (Senkowski et al., 2007). The subtraction metric led to four conditions: $\mathrm{PV}_{\mathrm{Fear}}$ minus $\mathrm{V}_{\mathrm{Fear}}, \mathrm{PV}_{\text {Anger }}$ minus $\mathrm{V}_{\text {Anger }}, \mathrm{PV}_{\text {Happiness }}$ minus $V_{\text {Happiness }}$, and $P V_{\text {Neutral }}$ minus $V_{\text {Neutral. }}$. Data from these conditions were subjected to repeated-measures ANOVA using the factors of facial expression (Fear, Anger, Happiness, and Neutral) and ROI (left sensorimotor hand region and right sensorimotor hand region). If significant effects of the factor facial expression were found, planned follow-up comparisons between the three emotional facial expression conditions and the neutral expression condition were computed. Additionally, it was examined whether the responses differed between the three emotional facial expressions using ANOVAs with the factors of facial expression (Fear, Anger, and Happiness) and ROI. Follow-up pairwise comparisons between the different emotion expression conditions, which were computed if a significant effect of facial expression was found in the ANOVA, were corrected for multiple testing using Bonferroni correction. Finally, if parallel effects were 
observed for the impact of facial expressions on pain ratings and paininduced oscillatory responses, Pearson correlation coefficients were computed for these effects across participants. Further details are provided in Results.

In the second level of analysis, the general effects of viewing faces, independent of their valence, on pain-induced responses were examined. To this end, oscillatory activity to P-only trials was compared with the activity to combined bimodal PV trials (averaged across the four conditions). The ANOVAs conducted comprised the factors of stimulation (P-only and averaged PV conditions) and ROI (left sensorimotor hand region and right sensorimotor hand region).

\section{Results}

\section{Pain ratings}

Since Shapiro-Wilk parametric tests, which were conducted within each participant and condition, revealed violations of normal distribution of non-catch trial pain ratings in the majority of tests ( $p<0.01$ in 46 of 72 tests), the median values of pain unpleasantness ratings were submitted to statistical analysis. Across participants and within conditions, no violations of normal distribution were found for the median values (none of the five conducted Shapiro-Wilk tests showed a $p<0.01$ ). Therefore, parametric tests were used to analyze the median values of pain ratings. The ANOVA for pain ratings to non-catch trials using the factor facial expression (Fear, Anger, Happiness, and Neutral) revealed a significant main effect $\left(F_{(3,17)}=3.31, p<0.05\right)$. Follow-up contrasts showed significantly higher pain ratings for all three emotional expressions than for the neutral expression condition (Fear vs Neutral: $F_{(1,17)}=6.14, p<0.05$; Anger vs Neutral: $F_{(1,17)}=4.5, p<0.05$; Happiness vs Neutral: $F_{(1,17)}=$ $6.33, p<0.05)$. Thus, the presentation of an emotional facial expression compared to the presentation of a neutral facial expression led to increased pain unpleasantness ratings (Fig. 1b). The comparisons between the three emotional facial expressions, however, did not reveal significant differences. Further analyses showed that the pain ratings for bimodal PV stimuli were for all conditions larger than the pain ratings for $\mathrm{P}$-only stimuli (all $p$ values $<0.0001)$. Finally, pain ratings in catch trials were significantly higher than pain ratings in non-catch trials (all $p$ values $<$ 0.0001; mean ratings for catch trials $=79$; data not shown), demonstrating that participants reliably rated the painful stimuli.

\section{Description of oscillatory response pattern}

Time-frequency representations $(2-120 \mathrm{~Hz})$ of pain-induced oscillatory responses showed early modulations in total GBA and BBA (Figs. 2, 3). At $\sim 170 \mathrm{~ms}$ after stimulus onset, an enhancement of GBA was observed, which was most pronounced contralateral to the intracutaneous stimulation site and at central sensors (Pattern I). Moreover, at the same latency an early suppression of BBA was observed (Pattern II). Although the duration of electrical stimuli was just $16 \mathrm{~ms}$, the very early $(<100 \mathrm{~ms})$ responses were contaminated by a broadband electrical stimulation artifact, which was strongest ipsilateral to the stimulation site. The artifact is smeared in time due to spectral smoothing in the time-frequency transformation but did not overlap with the time windows selected for the linear beamforming analysis. The observation that oscillatory GBA and BBA were predominantly observed in the sensorimotor hand region further suggests that the electromagnetic artifact did not contaminate the present findings (Fig. 2d).

\section{Effects of facial expressions on total beta-band activity}

A bilateral decrease of early BBA in the sensorimotor hand regions was found for all PV minus V conditions (Fig. 4). The
ANOVA to examine valence-specific effects on this early paininduced beta-band suppression (70-270 ms) using the factors of condition ( $P V_{\text {Fear }}$ minus $V_{\text {Fear }}, P_{\text {Anger }}$ minus $V_{\text {Anger }}, P V_{\text {Happiness }}$ minus $\mathrm{V}_{\text {Happiness }}$, and $P V_{\text {Neutral }}$ minus $\mathrm{V}_{\text {Neutral }}$ ) and ROI (left sensorimotor hand region and right sensorimotor hand region) revealed a significant main effect of facial expression $\left(F_{(3,51)}=4.45\right.$, $p<0.01)$. Follow-up contrasts between the three emotion conditions (separately) and the neutral expression condition using the factors of condition and ROI revealed significant main effects of the factor facial expression (Fear vs Neutral: $F_{(1,17)}=9.64, p<$ 0.01; Anger vs Neutral: $F_{(1,17)}=8.54, p<0.01$; Happiness vs Neutral: $\left.F_{(1,17)}=8.9, p<0.01\right)$. Thus, paralleling the findings of enhanced pain ratings for emotional compared to neutral facial expressions, the early BBA suppression was stronger in all three emotional expression conditions than in the neutral expression condition (Fig. 4b), while no significant differences were found between the three facial emotion conditions. No other main effects or interactions were found in these ANOVAs. Since the traces of sensors over contralateral sensorimotor regions indicated differences already at the onset of stimulation, oscillatory activity at -100 to $100 \mathrm{~ms}$ was analyzed in an additional ANOVA using the factors of condition and ROI. While this ANOVA revealed a significant main effect of ROI $\left(F_{(1,17)}=20.66, p<\right.$ $0.005)$ ), due to a larger contralateral than ipsilateral BBA, no significant effects were found in relation to the factor condition.

Next, it was examined across participants whether the impact of emotional facial expression on pain ratings, reflected by higher ratings to emotional than to neutral facial expressions, correlated with the effects on the pain-induced BBA. Although no significant correlation between pain ratings (mean of emotional expression conditions minus neutral condition) and BBA suppression (mean of emotional expression conditions minus neutral condition) was found for the left sensorimotor hand region (Pearson's $r=-0.23, p=0.35$ ), a significant positive correlation was found for the right sensorimotor hand region (Pearson's $r=0.51, p<$ 0.05). Participants with stronger effects of emotional facial expressions on pain ratings revealed larger differences in BBA between emotional and neutral facial expressions.

The general impact of faces on pain-induced BBA was examined in the second level of analysis in which responses to P-only trials were compared with averaged responses across PV conditions. The ANOVA for early BBA using the factors of stimulation (P-only and averaged PV conditions) and ROI (left sensorimotor hand region and right sensorimotor hand region) revealed a significant main effect of ROI, due to stronger amplitude suppression in the contralateral than in the ipsilateral region $\left(F_{(1,17)}=5.23, p<\right.$ 0.05). No other significant effects were found.

\section{Effects of facial expressions on total gamma-band activity}

The ANOVA to investigate valence-specific effects of facial expressions on early GBA using the factors of facial expression (Fear, Anger, Happiness, and Neutral) and ROI revealed a significant main effect of ROI $\left(F_{(3,51)}=11.87, p<0.005\right)$. The GBA was significantly larger in contralateral than in ipsilateral sensorimotor cortex (Fig. 5). No other significant effects were found. The ANOVA for the comparison between the GBA of the three emotional facial expression conditions using the factors of facial expression (Fear, Anger, and Happiness) and ROI revealed a significant main effect of facial expression $\left(F_{(2,34)}, p<0.05\right)$. Follow-up contrasts between all facial emotion expression pairs, revealed significantly larger pain-induced sensorimotor GBA for the facial expression of fear than for the expression of anger $\left(F_{(1,17)}=7.39, p<0.05\right.$, corrected for multiple testing $)$. Since no 
a

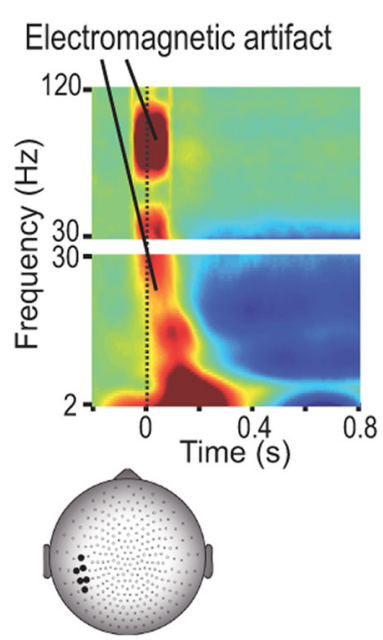

C

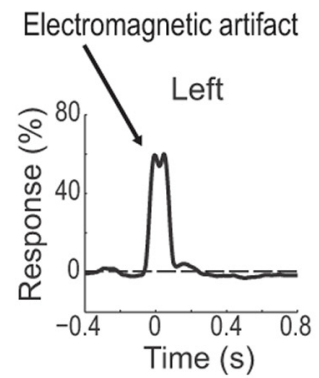

Electromagnetic artifact

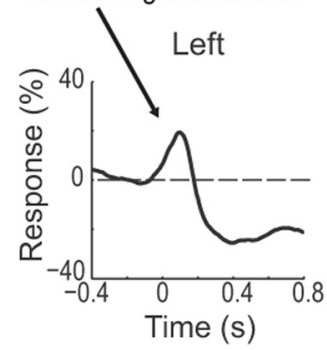

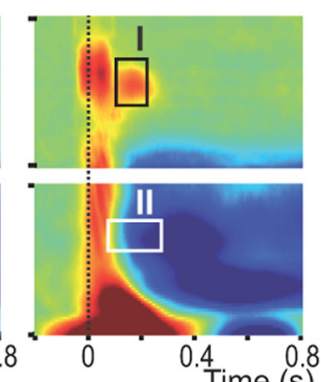

Time (s)
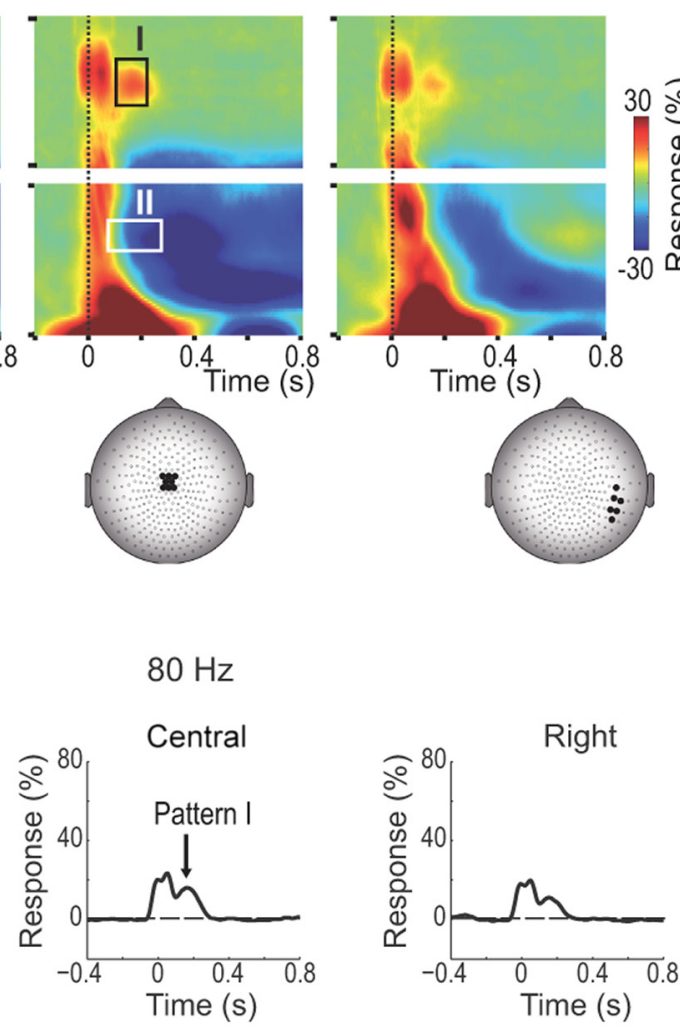

$18 \mathrm{~Hz}$

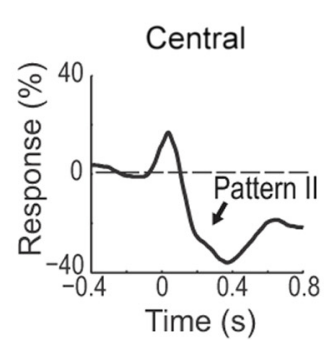

b
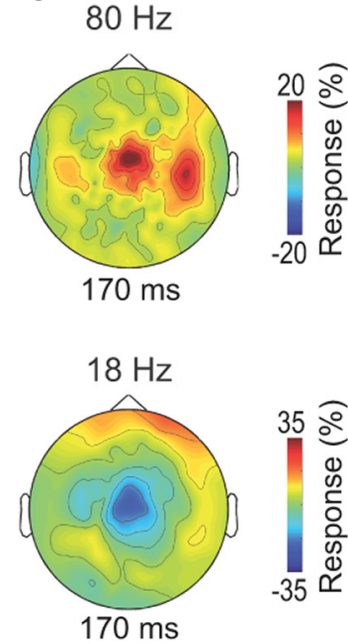

d

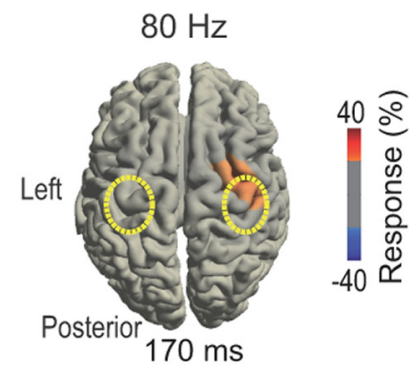

$18 \mathrm{~Hz}$

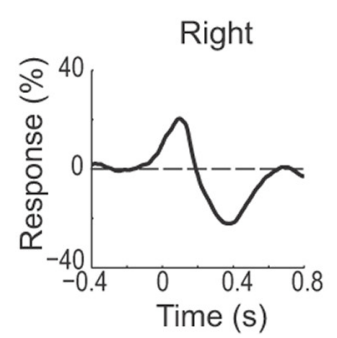

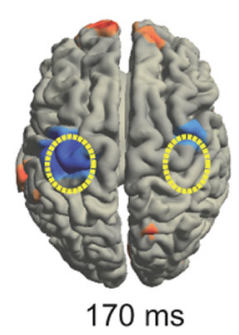

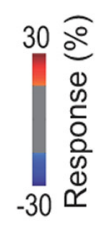

Figure 2. Total oscillatory MEG responses to pain-only stimuli. $\boldsymbol{a}$, Time-frequency representations for sensors located over sensorimotor areas (comprising 6 sensors each) and for central sensors (comprising 8 sensors). The selected sensors are highlighted in the head panels. Amplitude changes in early ( $\sim 170$ ms) oscillatory BBA and GBA were observed (highlighted by rectangles). The electromagnetic artifact induced by the intracutaneous stimulation contaminated the very early ( $<100 \mathrm{~ms}$ ) responses. $\boldsymbol{b}$, Topographic maps for GBA (upper panel) and BBA (lower panel). $\boldsymbol{c}$, Traces of GBA (upper panel) and BBA (lower panel). An increase in GBA was observed at $\sim 170$ ms (Pattern I). At the same latency, the BBA showed a decrease that was most pronounced at central sensors (Pattern II). $\boldsymbol{d}$, Results of the beamforming analysis of GBA (upper panel) and BBA (lower panel). Plotted are the highest and lowest quartiles of activity changes. A contralateral GBA increase was found that was paralleled by a bilateral BBA suppression.

differences in subjective ratings were found between the expressions of fear and anger, we did not compute a correlation analysis between pain ratings and the effects on GBA.

Next, the general effects of faces on the pain-induced GBA were examined. The ANOVA using the factors of stimulation (P-only vs combined PV) and ROI revealed a significant main effect of ROI $\left(F_{(1,17)}=24.78, p<0.0001\right)$, indicating larger amplitudes in the contralateral than in the ipsilateral ROI. Furthermore, a significant main effect of stimulation was found $\left(F_{(1,17)}=\right.$ 16.67, $p<0.001)$. The GBA was stronger in the combined PV conditions (average of left and right ROIs $=24 \%$ ) than in the P-only condition (average of left and right ROIs $=14 \%$ ). The stimulation $\times$ ROI interaction was not significant.
Effects of facial expressions on evoked oscillatory responses The study of differences between the four emotional facial expression conditions on early evoked BBA and GBA at the sensor level using the factors of facial expression (Fear, Anger, Happiness, and Neutral) and ROI (sensors located over left vs right sensorimotor regions) did not reveal any significant main effects or interactions. Furthermore, the sensor level ANOVAs examining the effects of facial expressions on the early evoked BBA by comparing P-only with combined PV trials did not reveal significant main effects or interactions. For the early evoked GBA, however, a significant main effect of stimulation was observed $\left(F_{(1,17)}=5.77, p<0.05\right)$, indicating stronger responses for combined PV than for P-only trials. 
a

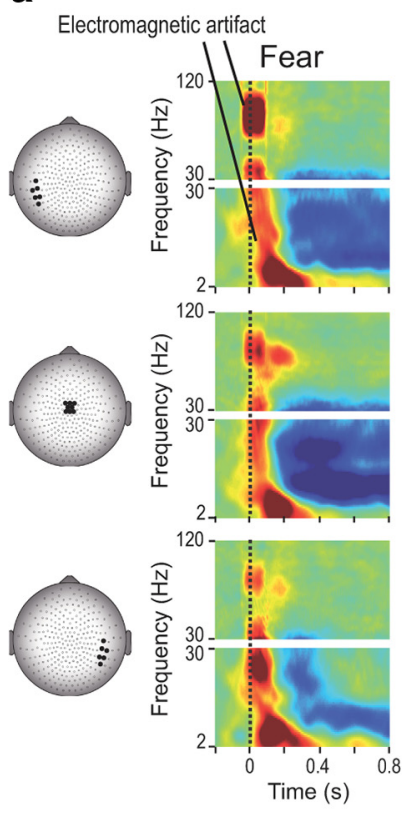

b
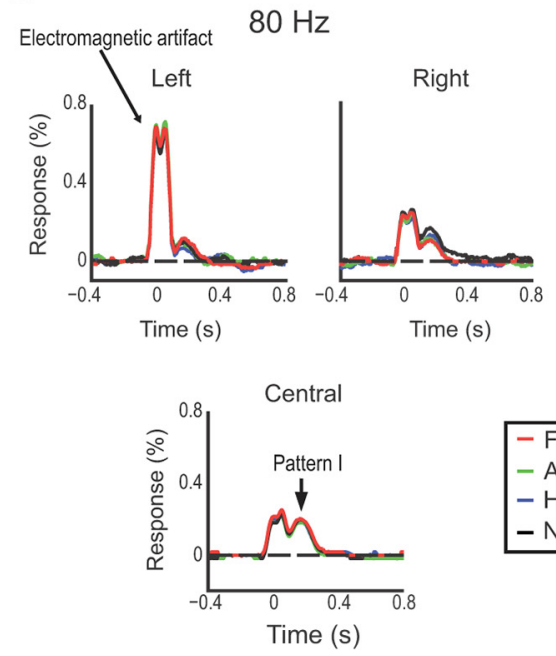

Bimodal PV minus V-only
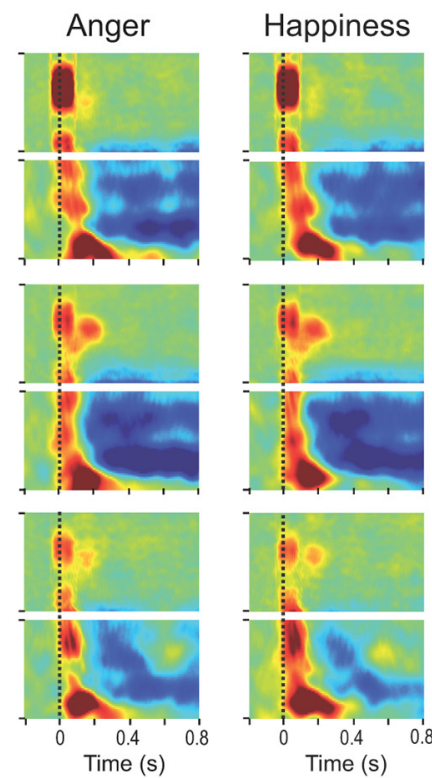

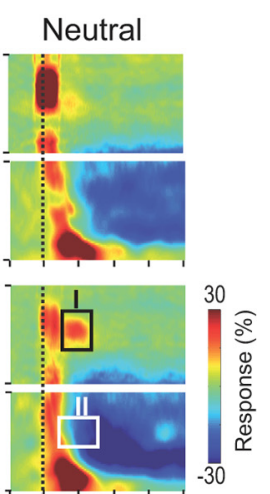

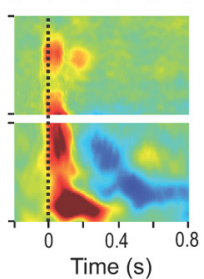
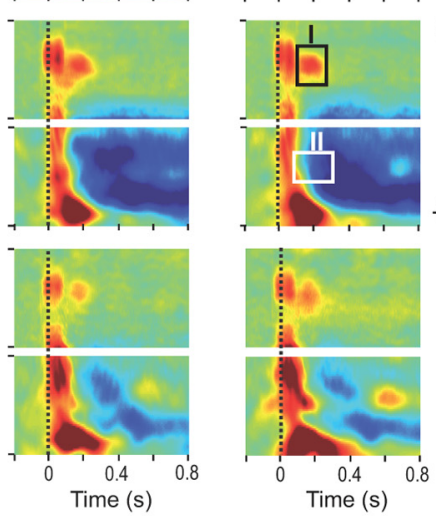

(20)
with pictures of positive valence (Meagher et al., 2001; Roy et al., 2009). The finding that emotional facial expressions lead to enhanced pain ratings independent of their valence may be linked to the important role of facial expression processing in the human brain, which involves a widespread network of cortical and subcortical structures (Adolphs, 2002; Calder and Young, 2005). Findings from patients with brain lesions (Adolphs et al., 2000), functional magnetic resonance imaging (Winston et al., 2003), and repetitive transcranial magnetic stimulation (Pitcher et al., 2008) demonstrated an involvement of right somatosensory cortex in the processing of emotional facial expression, which is widely independent of the valence of the expression. Thus, the presentation of emotional facial expressions in the present study may have facilitated the processing of painful stimuli in the somatosensory cortex, an assumption that is supported by our findings in oscillatory responses. Furthermore, it may be that painful inputs modulated the perception of happy faces. A recent study showed that painful inputs render pleasant pictures significantly less pleasant (Godinho et al., 2008). A biasing effect of painful stimuli on inputs with positive valence from other sensory modalities may have also contributed to the observation that a lemon scent odor, which was rated as pleasant when presented alone, enhanced pain perception (Martin, 2006). It can be

\section{Discussion}

This MEG study examined the impact of emotional facial expressions on pain perception and pain processing in the human brain. We observed that the presentation of faces expressing fear, anger, and happiness compared to the presentation of neutral facial expressions led to an increase in subjective pain ratings. Emotional facial expression had a modulatory effect on early oscillatory beta- and gamma-band responses in the sensorimotor cortex.

\section{Pain ratings}

Our study revealed that facial expressions with negative and positive valence enhance subjective pain unpleasantness ratings

speculated that a happy face presented in combination with a painful stimulus may be interpreted as if this person is laughing about oneself. While the present study focused on the impact of viewing faces with emotional expressions on pain processing, future studies may address how painful inputs modulate the perceived valence and arousal of facial expressions.

In the present study, participants fixated on the faces without an explicit task and rated the unpleasantness of concurrently presented painful inputs. Previous studies showed that in the absence of a demanding secondary task (Pessoa et al., 2002), the decoding of facial expressions occurs rapidly and widely automatically (Vuilleumier et al., 2001; Eimer and Holmes, 2007). Therefore, it is likely that the emotional facial expressions were 
a

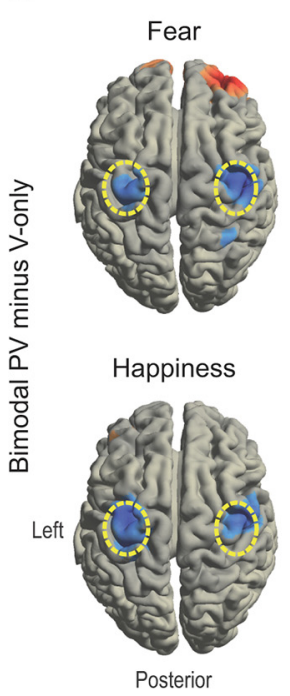

$18 \mathrm{~Hz}, 170 \mathrm{~ms}$

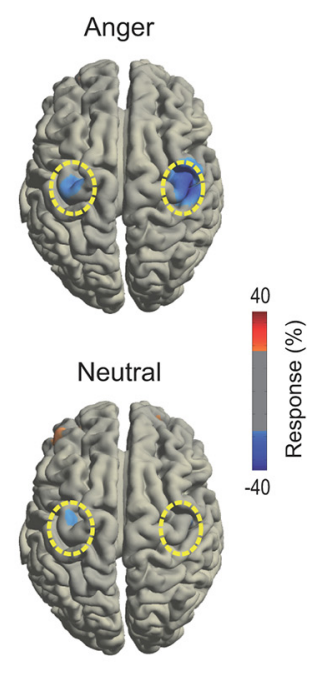

b Sensorimotor hand region, $18 \mathrm{~Hz}, 170 \mathrm{~ms}$

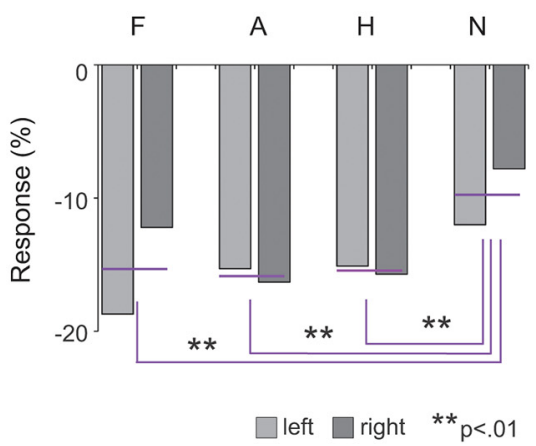

Figure 4. Beamforming analysis of early beta-band responses for the contrasts of bimodal PV and the corresponding V-only stimuli. $\boldsymbol{a}$, Robust pattern of BBA modulations at the sensorimotor hand region (highlighted by dotted circles) were found. Plotted are the highest and lowest quartiles of activity changes. $\boldsymbol{b}$, The statistical analysis of BBA at the left and right sensorimotor hand region (bimodal PV minus visual-only) revealed significantly lower betaband responses for the three emotional facial expression conditions [i.e., fear ( $\mathrm{F})$, anger (A), and happiness $(\mathrm{H})$ ] than for the neutral $(\mathrm{N})$ expression condition. Horizontal lines are plotted to illustrate the mean values of BBA across the left and right sensorimotor hand regions.

automatically processed. Our study showed that viewing faces with both positive and negative valence enhances subjective pain perception compared to viewing faces with neutral expressions.

\section{Pain-induced beta-band responses}

In line with previous studies using tactile or painful stimuli (Salenius et al., 1997; Bauer et al., 2006; Hauck et al., 2007), the suppression of beta-band responses was localized to the sensorimotor cortex. Due to the widespread distribution of the BBA suppression, we cannot disentangle whether the reported effects mostly reflect processing in the motor cortex or processing in somatosensory areas. Previous studies showed that nociceptive stimuli cause a reduction of ongoing $\sim 20 \mathrm{~Hz}$ oscillations both in the primary motor cortex (Raij et al., 2004) and in somatosensory areas (Ploner et al., 2006a). Thus, it may be that the sensorimotor BBA reflect parallel processing in somatosensory and motor areas, which form a functional network through coupling of neuronal populations (Schnitzler et al., 2000; Brovelli et al., 2002). Notably, beta-band responses have been suggested to modulate the excitability of the sensorimotor cortex. Ploner et al. (2006b) showed that pain-induced suppression of BBA is positively cor-

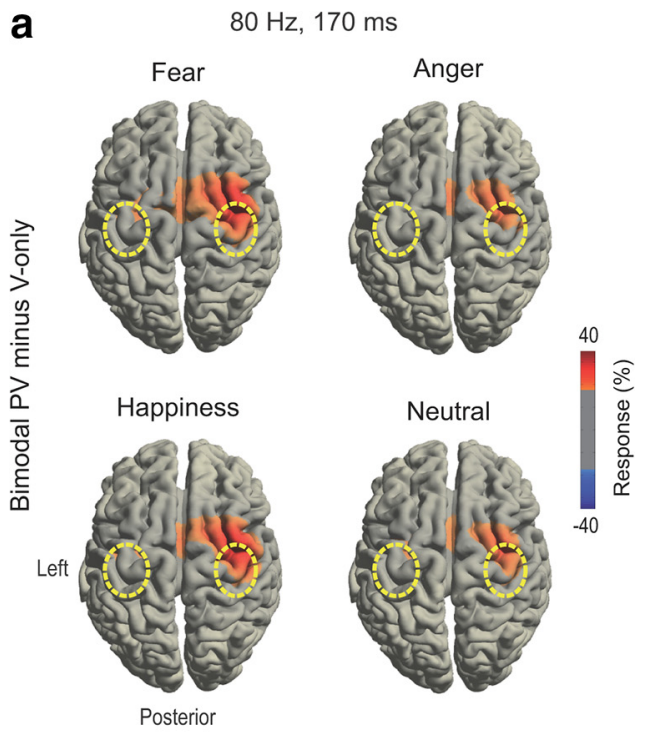

b Sensorimotor hand region, $80 \mathrm{~Hz}, 170 \mathrm{~ms}$

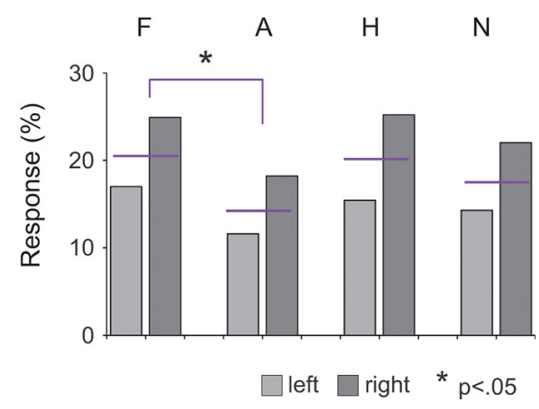

Figure 5. Beamforming analysis of total gamma-band responses for the contrasts of bimodal PV and the corresponding V-only stimuli. $\boldsymbol{a}$, A robust increase in GBA was found at the right sensorimotor hand region at a short latency of $170 \mathrm{~ms}$ (left and right sensorimotor hand regions are highlighted by dotted circles). Plotted are the highest and lowest quartiles of activity changes. $\boldsymbol{b}$, The statistical analysis of GBA (bimodal PV minus visual only) at the left and right sensorimotor hand region revealed significantly higher responses for the facial expression fear than for the facial expression anger but no significant differences between the three emotional facial expression conditions and the neutral expression condition. Horizontal lines are plotted to illustrate the mean values of GBA across the left and right sensorimotor hand regions.

related with the excitability of the sensorimotor system. In the present study, the enhanced early suppression of BBA for emotional compared to neutral facial expressions therefore suggests that facial expressions, which represent particularly powerful emotional cues, enhance the sensitivity of pain processing in the sensorimotor cortex.

The strongest pain-induced responses in our study were found in areas of the sensorimotor cortex. Pain processing in sensorimotor cortex primarily reflects the sensory-discriminative dimension of the nociceptive perception, which encompass the perceived intensity, location, and quality of painful events (Bushnell et al., 1999; Lorenz et al., 2005). Therefore, it is likely that participants incorporated the perceived intensity, which has not been separately monitored, in their pain ratings. The observation that pain-induced responses were reflected in early oscillatory responses in the beta- and gamma-band that were localized to the sensorimotor cortex supports this assumption. Together, our data suggest that emotional facial expressions, independent of their valence, increase the sensitivity of pain processing in the sensorimotor cortex through an enhanced suppression of the early pain-induced BBA. Since BBA suppression in the sensorimotor cortex has also been closely associated with the prepara- 
tion of motor responses (Pfurtscheller and Lopes da Silva, 1999; Neuper et al., 2006), our data indicate that the concurrent presentation of an emotional facial expression while receiving a painful input may facilitate the preparation and execution of adequate protective motor responses.

Another interesting finding is that the effects of emotional facial expressions on subjective pain ratings were correlated in particular with the BBA modulations in the contralateral sensorimotor cortex. This suggests that the observed effects of emotional facial expression on the BBA most likely reflect pain-related processing and not the preparation of the motor response that was required for the rating of the painful stimulus. Since participants rated painful inputs with a joystick in their right hand, confounding effects of motor preparation on oscillatory responses would be expected to occur primarily in the left sensorimotor cortex.

\section{Pain-induced gamma-band responses}

In contrast to the BBA, the pain-induced GBA did not differ significantly between the neutral and emotional facial expression conditions. However, the direct comparison of the pain-induced GBA between the different emotion conditions revealed larger responses to faces expressing fear than to faces expressing anger. While both emotions have a negative valence, this finding is noteworthy when considering approach-related models for the processing of anger (Carver and Harmon-Jones, 2009). Recent studies demonstrated that the presentation of angry facial expressions facilitates approach-motivated motor behavior toward angry faces (Wilkowski and Meier, 2010). Angry facial expressions may communicate an intention to confront another person aggressively, thereby facilitating approach-motivated rather than avoidance-motivated behavior. By contrast, acute pain primarily triggers avoidance-motivated behavior (Bromm and Lorenz, 1998). Since the sensorimotor GBA has been, among other functions, linked to the initiation of a motor response (Crone et al., 1998), it can be speculated that the reduced GBA in the sensorimotor cortex may represent the conflict between approachmotivated behavior initiated by the angry facial expressions and avoidance-motivated behavior initiated by the painful inputs. Our observation that the strongest differences in pain-induced GBA were found between fearful and angry facial expressions fits with this interpretation. Similar to painful stimuli, fearful facial expressions have been associated with avoidance-motivated behavior (Davidson, 1998).

Factors that may have contributed to the reported effects of facial expressions on pain-induced oscillatory responses are arousal and attention (Anders et al., 2004; Wiech et al., 2008). Emotional arousal is reflected in modulations of neuronal synchronization across different frequency ranges (Keil et al., 2001; Steriade, 2004). Moreover, functional magnetic resonance studies have shown that attention is positively associated with pain perception and activity of nodes of the pain matrix (Villemure et al., 2009; Ploner et al., 2011). Since attention and arousal have not been explicitly monitored in the present study, it remains to be elucidated to what extent these factors may contribute to the impact of emotional facial expressions on pain processing.

\section{Conclusion}

Our study revealed several important findings. Compared to neutral facial expressions, the presentation of emotional facial expressions enhanced the perception of pain. Moreover, we found that both early BBA and GBA in the sensorimotor cortex were modulated by emotional facial expressions. Finally, the observation of a relationship between the effects on early
BBA in the sensorimotor cortex and the effects on subjective pain ratings suggests an involvement of pain-induced oscillatory responses in the emotional modulation of pain processing and pain perception.

\section{Notes}

Supplemental material for this article is available at http://www.uke. de/institute/neurophysiologie/downloads/institut-neurophysiologiepathophysiologie/Senkowski_2011_JN_Additional_Material.doc and shows effects of emotional facial expressions on pain-induced lower-frequency (3-9 Hz) activity. This material has not been peer reviewed.

\section{References}

Adolphs R (2002) Neural systems for recognizing emotion. Curr Opin Neurobiol 12:169-177.

Adolphs R, Damasio H, Tranel D, Cooper G, Damasio AR (2000) A role for somatosensory cortices in the visual recognition of emotion as revealed by three-dimensional lesion mapping. J Neurosci 20:2683-2690.

Anders S, Lotze M, Erb M, Grodd W, Birbaumer N (2004) Brain activity underlying emotional valence and arousal: a response-related fMRI study. Hum Br Mapp 23:200-209.

Batty M, Taylor MJ (2003) Early processing of the six basic facial emotional expressions. Brain Res Cogn Brain Res 17:613-620.

Bauer M, Oostenveld R, Peeters M, Fries P (2006) Tactile spatial attention enhances gamma-band activity in somatosensory cortex and reduces lowfrequency activity in parieto-occipital areas. J Neurosci 26:490-501.

Bromm B, Lorenz J (1998) Neurophysiological evaluation of pain. Electroencephalogr Clin Neurophysiol 107:227-253.

Bromm B, Meier W (1984) The intracutaneous stimulus: a new pain model for algesimetric studies. Methods Find Exp Clin Pharmacol 6:405-410.

Brovelli A, Battaglini PP, Naranjo JR, Budai R (2002) Medium-range oscillatory network and the $20-\mathrm{Hz}$ sensorimotor induced potential. Neuroimage 16:130-141.

Bushnell MC, Duncan GH, Hofbauer RK, Ha B, Chen JI, Carrier B (1999) Pain perception: is there a role for primary somatosensory cortex? Proc Natl Acad Sci U S A 96:7705-7709.

Calder AJ, Young AW (2005) Understanding the recognition of facial identity and facial expression. Nat Rev Neurosci 6:641-651.

Carver CS, Harmon-Jones E (2009) Anger is an approach-related affect: evidence and implications. Psychol Bull 135:183-204.

Crone NE, Miglioretti DL, Gordon B, Lesser RP (1998) Functional mapping of human sensorimotor cortex with electrocorticographic spectral analysis. II. Event-related synchronization in the gamma band. Brain 121:2301-2315.

Davidson RJ (1998) Affective style and affective disorders: perspectives from affective neuroscience. Cogn Emot 12:307-330.

Donner TH, Siegel M, Fries P, Engel AK (2009) Buildup of choicepredictive activity in human motor cortex during perceptual decision making. Curr Biol 19:1581-1585.

Eimer M, Holmes A (2007) Event-related brain potential correlates of emotional face processing. Neuropsychologia 45:15-31.

Frith C (2009) Role of facial expressions in social interactions. Philos Trans R Soc Lond B Biol Sci 364:3453-3458.

Godinho F, Magnin M, Frot M, Perchet C, Garcia-Larrea L (2006) Emotional modulation of pain: is it the sensation or what we recall? J Neurosci 26:11454-11461.

Godinho F, Frot M, Perchet C, Magnin M, Garcia-Larrea L (2008) Pain influences hedonic assessment of visual inputs. Eur J Neurosci 27:2219-2228.

Gross J, Kujala J, Hamalainen M, Timmermann L, Schnitzler A, Salmelin R (2001) Dynamic imaging of coherent sources: studying neural interactions in the human brain. Proc Natl Acad Sci U S A 98:694-699.

Gross J, Schnitzler A, Timmermann L, Ploner M (2007) Gamma oscillations in human primary somatosensory cortex reflect pain perception. PLoS Biol 5:e133.

Hauck M, Lorenz J, Engel AK (2007) Attention to painful stimulation enhances gamma-band activity and synchronization in human sensorimotor cortex. J Neurosci 27:9270-9277.

Hauck M, Lorenz J, Engel AK (2008) Role of synchronized oscillatory brain activity for human pain perception. Rev Neurosci 19:441-450.

Kayser C, Logothetis NK (2009) Directed interactions between auditory and 
superior temporal cortices and their role in sensory integration. Front Integr Neurosci 3:7.

Keil A, Müller MM, Gruber T, Wienbruch C, Stolarova M, Elbert T (2001) Effects of emotional arousal in the cerebral hemispheres: a study of oscillatory brain activity and event-related potentials. Clin Neurophysiol 112:2057-2068.

Knosche TR (2002) Transformation of whole-head MEG recordings between different sensor positions. Biomed Tech (Berl) 47:59-62.

Lakatos P, Chen CM, O'Connell MN, Mills A, Schroeder CE (2007) Neuronal oscillations and multisensory interaction in primary auditory cortex. Neuron 53:279-292.

Lorenz J, Hauck M, Paur RC, Nakamura Y, Zimmermann R, Bromm B, Engel AK (2005) Cortical correlates of false expectations during pain intensity judgments-a possible manifestation of placebo/nocebo cognitions. Brain Behav Immun 19:283-295.

Martin GN (2006) The effect of exposure to odor on the perception of pain. Psychosom Med 68:613-616.

Meagher MW, Arnau RC, Rhudy JL (2001) Pain and emotion: effects of affective picture modulation. Psychosom Med 63:79-90.

Mitra PP, Pesaran B (1999) Analysis of dynamic brain imaging data. Biophys J 76:691-708.

Nakata H, Tamura Y, Sakamoto K, Akatsuka K, Hirai M, Inui K, Hoshiyama M, Saitoh Y, Yamamoto T, Katayama Y, Kakigi R (2008) Evoked magnetic fields following noxious laser stimulation of the thigh in humans. Neuroimage 42:858-868.

Neuper C, Wörtz M, Pfurtscheller G (2006) ERD/ERS patterns reflecting sensorimotor activation and deactivation. Prog Brain Res 159:211-222.

Pessoa L, McKenna M, Gutierrez E, Ungerleider LG (2002) Neural processing of emotional faces requires attention. Proc Natl Acad Sci U S A 99:11458-11463.

Pfurtscheller G, Lopes da Silva FH (1999) Event-related synchronization and desynchronization: basic principles. Clin Neurophysiol 110:1842-1857.

Pitcher D, Garrido L, Walsh V, Duchaine BC (2008) Transcranial magnetic stimulation disrupts the perception and embodiment of facial expressions. J Neurosci 28:8929-8933.

Ploner M, Schmitz F, Freund HJ, Schnitzler A (1999) Parallel activation of primary and secondary somatosensory cortices in human pain processing. J Neurophysiol 81:3100-3104.

Ploner M, Gross J, Timmermann L, Pollok B, Schnitzler A (2006a) Pain suppresses spontaneous brain rhythms. Cereb Cortex 16:537-540.

Ploner M, Gross J, Timmermann L, Pollok B, Schnitzler A (2006b) Oscillatory activity reflects the excitability of the human somatosensory system. Neuroimage 32:1231-1236.

Ploner M, Lee MC, Wiech K, Bingel U, Tracey I (2011) Flexible cerebral connectivity patterns subserve contextual modulations of pain. Cereb Cortex 21:719-726.

Price DD (2000) Psychological and neural mechanisms of the affective dimension of pain. Science 288:1769-1772.

Raij TT, Forss N, Stancák A, Hari R (2004) Modulation of motor-cortex oscillatory activity by painful Adelta- and C-fiber stimuli. Neuroimage 23:569-573.

Rhudy JL, Bartley EJ, Williams AE (2010) Habituation, sensitization, and emotional valence modulation of pain responses. Pain 148:320-327.

Roy M, Piché M, Chen JI, Peretz I, Rainville P (2009) Cerebral and spinal modulation of pain by emotions. Proc Natl Acad Sci U S A 106:20900-20905.

Salenius S, Schnitzler A, Salmelin R, Jousmäki V, Hari R (1997) Modulation of human cortical rolandic rhythms during natural sensorimotor tasks. Neuroimage 5:221-228.

Schneider TR, Debener S, Oostenveld R, Engel AK (2008) Enhanced EEG gamma-band activity reflects multisensory semantic matching in visualto-auditory object priming. Neuroimage 42:1244-1254.

Schneider TR, Lorenz S, Senkowski D, Engel AK (2011) Gamma-band activity as a signature for cross-modal priming of auditory object recognition by active haptic exploration. J Neurosci 31:2502-2510.

Schnitzler A, Gross J, Timmermann L (2000) Synchronised oscillations of the human sensorimotor cortex. Acta Neurobiol Exp (Wars) 60:271-287.

Senkowski D, Molholm S, Gomez-Ramirez M, Foxe JJ (2006) Oscillatory beta activity predicts response speed during a multisensory audiovisual reaction time task: a high-density electrical mapping study. Cereb Cortex 16:1556-1565.

Senkowski D, Gomez-Ramirez M, Lakatos P, Wylie GR, Molholm S, Schroeder CE, Foxe JJ (2007) Multisensory processing and oscillatory activity: analyzing non-linear electrophysiological measures in humans and simians. Exp Brain Res 177:184-195.

Senkowski D, Schneider TR, Foxe JJ, Engel AK (2008) Crossmodal binding through neural coherence: implications for multisensory processing. Trends Neurosci 31:401-409.

Steriade M (2004) Acetylcholine systems and rhythmic activities during the waking-sleep cycle. Prog Brain Res 145:179-196.

Tiemann L, Schulz E, Gross J, Ploner M (2010) Gamma oscillations as a neuronal correlate of the attentional effects of pain. Pain 150:302-308.

Van Veen BD, van Drongelen W, Yuchtman M, Suzuki A (1997) Localization of brain electrical activity via linearly constrained minimum variance spatial filtering. IEEE Trans Biomed Eng 44:867-880.

Villemure C, Bushnell MC (2009) Mood influences supraspinal pain processing separately from attention. J Neurosci 29:705-715.

Vuilleumier P, Armony JL, Driver J, Dolan RJ (2001) Effects of attention and emotion on face processing in the human brain: an event-related fMRI study. Neuron 30:829-841.

Wiech K, Ploner M, Tracey I (2008) Neurocognitive aspects of pain perception. Trends Cogn Sci 12:306-313.

Wilkowski BM, Meier BP (2010) Bring it on: angry facial expressions potentiate approach-motivated motor behavior. J Pers Soc Psychol 98:201-210.

Winston JS, O’Doherty J, Dolan RJ (2003) Common and distinct neural responses during direct and incidental processing of multiple facial emotions. Neuroimage 20:84-97.

Young AW, Perrett D, Calder A, Sprengelmeyer R, Ekman P (2002) Facial expressions of emotions: stimuli and test (FEEST). Thurstone, UK: Thames Valley Test Company. 